\title{
Représentations de la sagesse dans le livre de Baruch
}

\author{
Elena Di PEDE \\ (Université de Lorraine-Metz -EA 3943, Centre de recherche Écritures)
}

Le texte de $\mathrm{Ba}$ 3,9-4,4 est une belle et longue exhortation à la sagesse qui a déjà été amplement travaillée pour en déterminer les liens avec le Deutéronome et les livres de Sagesse (Job 28 et Si 24 en particulier ${ }^{1}$ ). Des livres de Sagesse cette exhortation reprend thèmes et vocabulaire, du Deutéronome elle reprend l'importance et la centralité de la Torah, au point d'identifier Torah et sagesse.

Je voudrais consacrer cette contribution à deux aspects : dans un premier temps, je proposerai un commentaire rapide de ce poème. Dans un second temps, je voudrais regarder plus spécifiquement comment cette exhortation s'insère dans la littérature jérémienne $\mathrm{LXX}^{2}$, et en particulier avec Jérémie qui précède Baruch et qui est le seul de cet ensemble jérémien à utiliser la vaste gamme des termes qui touchent à la sagesse. Les termes tels que sophia et ses apparentés, verbes et substantifs (sophos, sophôs, sophistês, sophoô), phronêsis (c'est-à-dire la sagesse pratique), qui est pour certains synonyme de sophia $a^{3}$ on reviendra plus avant sur la distinction entre les deux termes - et ses apparentés, verbes et substantifs (phronêsis, phronế, phronêma), mais aussi gnôsis, dianoia, sunetos, sunetôs et sunesis, nomizo (penser), oida, epistamai (être savant) et epistêmê (science) ${ }^{4}$ - j'en oublie certainement - tous ces termes créent des liens et des résonnances qui me semblent intéressants entre Jérémie et Baruch ${ }^{5}$.

1. Outre les commentaires, on verra par exemple Burkes 1999 (http://www.jstor.org/stable/ 24668456) ; voir aussi Kabasele Mukenge 1998, p. 254-276.

2. Baruch étant un livret deutérocanonique, il est méthodologiquement important de se limiter au corpus grec.

3. En ce sens, par exemple, Assan-Dhôte, Moatti-Fine 2008 et Kabasele Mukenge 1998 qui considère que tout ce vocabulaire "ne semble pas introduire des nuances particulières ", p. 229.

4. À ce panorama, on pourrait ajouter les antonymes, comme aboulia par exemple. Celui-ci n'intervient qu'une seule fois en $\mathrm{Ba}(3,28)$. On le retrouve ailleurs uniquement en Pr 14,17.

5. Notons d'emblée que le vocabulaire relevé concerne - sauf erreur de ma part - massivement Jr et Ba et beaucoup moins LtJr ou Lm, les deux autres textes que la LXX place sous l'égide de Jérémie. Ainsi, l'utilisation de ce vocabulaire spécifique semble confirmer la très grande familiarité entre ces deux écrits. Je rappelle que, pour bon nombre des Pères

Représentations et personnification de la sagesse dans l'Antiquité et au-delà, sous la direction de Stéphanie Anthonioz et Cécile Dogniez, 2021 - p. 195-206 


\section{L'EXHORTATION À LA SAGESSE DANS BARUCH (BA 3,9-4,4)}

Ce poème est central dans Baruch, enchâssé qu'il est entre la prière de pénitence (Ba 1,15-3,8) auquel en quelque sorte il répond, et la promesse de salut (Ba 4,5-5,9). Cette disposition semble assez logique : la promesse de salut ne peut venir qu'après la pénitence proclamée et assumée, alors que la nouvelle orientation donnée au peuple dans cette exhortation apparaît également comme une sorte de recadrage quant à sa mission (cf. 4,2-4). Avant d'aller plus loin il n'est probablement pas inutile de lire le texte ${ }^{6}$, ici proposé de manière à mettre en évidence quelques éléments structurels.

$3,{ }^{9}$ ÉCoute, Israël, les commandements de vie, prêtez l'oreille pour connaître la sagesse-pratique (phronêsis).

10 Qu'y a-t-il, Israël, qu'y a-t-il pour que tu sois dans le pays des ennemis, que tu aies vieilli dans un pays étranger,

11 que tu aies été souillé avec les cadavres et compté parmi ceux de l'Hadès?

12 Tu as abandonné la source de la sagesse (sophia).

13 Si tu avais suivi la route de Dieu, tu habiterais dans la paix pour toujours.

14 APPRENDS où est la sagesse-pratique (phronêsis), où est la force, où est l'intelligence pour connaître en même temps où est la longévité et la vie, où est la lumière des yeux et la paix. Qui a découvert son lieu et qui a pénétré ses ${ }^{7}$ trésors?

16 Où sont les princes des Nations et les maîtres des bêtes qui sont sur la terre,

17 et ceux qui se jouent des oiseaux du ciel, qui thésaurisent l'argent et l'or, en quoi se sont fiés les humains, sans qu'il y ait de limite à leur possession,

mais la route de la science, ils ne l'ont pas connue,

21 ils n'ont pas compris ses chemins, ils ne l'ont pas saisie/gagnée ;

et leurs fils se sont éloignés de leur route.

22 Elle n'a pas été entendue en Canaan ni vue en Témân ;

23 et les fils d'Agar qui cherchaient l'intelligence sur la terre,

les marchands de Merrân et de Témân, les faiseurs de contes et les chercheurs d'intelligence n'ont pas connu pourtant la route de la sagesse (sophia) ni ne se sont souvenus de ses chemins.

Ô Israël ! Comme elle est grande la maison de Dieu, étendu le lieu de sa possession ;

de l'Église, ces deux écrits sont considérés comme un seul et même livre ; du reste, ils attribuent à Jérémie des passages situés en $\mathrm{Ba}$. On verra à ce propos les travaux de Pierre-Maurice Bogaert, en particulier 1974 et 1982.

6. La traduction ici proposée est celle de Assan-Dhôte, Moatti-Fine 2008, quelque peu adaptée, notamment sur la différence entre les termes sophia et phronêsis. On verra aussi Kabasele Mukenge 1998, en particulier p. 221-224 pour la traduction et p. 225-283 pour le commentaire.

7. Logiquement le substantif féminin antécédent du pronom est phronêsis (cf. v. 14). Mais le pronom ne pourrait-il pas aussi renvoyer à sophia? 

et la Loi (nomos, m.) qui existe pour toujours ; tous ceux qui la ${ }^{10}$ tiennent seront pour la vie, mais ceux qui la délaissent mourront.

2 Retourne / Reviens, Jacob, et saisis-toi d'elle ${ }^{11}$, mets-toi en route vers la clarté en face de sa lumière ;

3 ne donne pas à un autre ta gloire ni ce qui t'est utile / tes avantages à une nation étrangère.

4 Heureux sommes-nous, Israël, parce que ce qui plaît à Dieu nous est connu.

D'après Walter Harrelson ${ }^{12}$, ce poème rassemble et tisse cinq thématiques bibliques, dont seule la première lui semble originale. Je les reprends en les discutant quelque peu :

1. Israël est en exil parce qu'il n'a pas acquis la sagesse. Cela semble évident dans l'argumentation de l'exhortation. On peut cependant ajouter à cela l'insistance forte sur le refus d'écouter la Parole, élément presque martelé au début du poème et très présent dans la première partie de Baruch. Ainsi l'exhortation semble suggérer un lien fort entre le refus d'écouter et la non-acquisition de la sagesse ${ }^{13}$.

8. Logiquement le groupe nominal féminin antécédent du pronom est hodos epistêmês (la route de la science, v. 37). Mais cela pourrait aussi renvoyer à phronêsis ou à sophia.

9. Voir note 8.

10. Voir note 8.

11. Voir note 8.

12. Voir Harrelson 1992, p. 158-171.

13. Cet élément crée un lien thématique fort avec Jérémie où la question du refus d'écoute du peuple, cause de son départ en exil, est centrale. 
2. Aucun humain ne peut trouver la sagesse à lui seul. Cet élément crée une opposition, d'une part entre Dieu et les humains, et d'autre part, chez les humains, entre Jacob / Israël et les Nations ; en effet, Dieu a donné la sagesse au peuple qu'il a choisi et non aux autres. Cela dit, cette question demeure quelque peu ambiguë dans le texte car un flou est entretenu sur le type de sagesse dont il est question : s'agit-il de la phronêsis, de la sophia ou encore de la hodos epistêmês? D'autant plus que les trois termes sont féminins. Dès lors, auquel des deux premiers et parfois des trois se réfère le pronom personnel de $3^{\mathrm{e}}$ personne du féminin ? Ainsi la question qui se pose est celle de comprendre de quelle sagesse il s'agit $\mathrm{ici}^{14}$. On reviendra sur ce point.

3. La sagesse est présente à la création (v. 32-34), un thème qui se trouve dans d'autres livres sapientiaux (cf. Pr 8,22-31).

4. Dieu a donné la sagesse à Israël (cf. v. 37 : en lien avec la route de la science). Cette thématique est clairement liée à la question de l'élection. Cela dit, dans notre exhortation, la question pourrait être de savoir laquelle des formes de sagesse Israël a reçue.

5. La sagesse - mais laquelle ? - est identifiée à la Torah.

Toujours selon Harrelson, ce poème poursuit un double but: en premier lieu souligner la nécessité de reconnaître la Torah comme unique source d'orientation vitale pour la communauté ; ensuite identifier la Torah à la sagesse : la Torah c'est la sagesse et la sagesse c'est la Torah ${ }^{15}$.

Tel qu'il se présente au lecteur, le poème se divise en trois parties qui commencent toutes trois par un impératif adressé à Jacob / Israël - qui est différemment désigné à chaque entame de partie : Israël en 3,9; sous-entendu en 3,14; Jacob en 4,2. Cet impératif lui enjoint d'accomplir une action fondamentale en lien avec sa vie, actuelle ou future : écouter et prêter l'oreille, apprendre, revenir. Il en ressort que l'ensemble vise à convaincre Israël « de la nullité des sagesses autres que celle que Dieu a montrée à travers la Loi écrite ${ }^{16}$, afin que le peuple adopte cette attitude fondamentale d'écoute de la parole divine et que, ainsi, il vive en alliance.

\section{Première partie : 3,9-13}

La première partie commence par un impératif présent actif du verbe akouô, "écouter», adressé à Israël ; il est redoublé par l'impératif aoriste de enôtizomai, "prêter l'oreille», «faire attention à », adressé à un « vous », qui peut viser le peuple collectivement et individuellement. Ainsi à ce double niveau - individuel et collectif -, Israël est pris à partie par le biais d'une insistance sur l'écoute. Cela montre l'importance, mais aussi l'urgence de cette action à poser car, en terre étrangère, Israël doit

14. Pour reprendre les termes utilisés par Christian Cannuyer lors de la journée d'étude, à propos de la Maât, une forme de sagesse serait "cosmique», l'autre plus «sociale», et se déclinerait sur le plan pratique et éthique.

15. Selon Harrelson, cette identification attire l'attention sur le fait qu'il est impossible de percer entièrement les secrets de l'une ou de l'autre. Cela implique un acte d'interprétation qui n'est jamais terminé car Dieu seul connaît en plénitude la Torah et la sagesse. Sur l'identification entre Torah et sagesse, voir aussi Kabasele Mukenge 1998, p. 267-268.

16. La structure ainsi proposée diffère légèrement de celle de Kabasele Mukenge 1998, p. 232-233, par exemple, qui considère que la $2^{e}$ partie du poème commence en $3,15$. 
comprendre les raisons de cet exil et s'éloigner de ce qui le détourne de la sagesse (phronêsis), comme l'indique la fin du poème $(4,2)$. D'emblée, ce parallèle entre les impératifs initiaux suggère la correspondance entre les commandements à suivre pour avoir la vie et la sagesse (phronêsis), une correspondance qui sera reprise dans la deuxième partie, tant au début $(3,14)$ qu'à la fin où se fera l'identification claire entre Torah et sagesse $(4,1)$.

L'appel à l'écoute s'adresse donc à Israël en terre étrangère $(3,10)$ et semble répondre aux trois interpellations directes destinées à Dieu dans la prière de repentance collective (cf. 2,4 : impératif aoriste de eisakouô ; 3,2.4 : impératif aoriste de akouô). Cet appel est solennel, comme il se doit pour une exhortation qui invite le peuple à changer de comportement, afin d'acquérir la sagesse et de réapprendre à cheminer vers Dieu, source de sophia (cf. 3,12). Pour Baruch les choses sont claires : si le peuple avait agi comme il lui avait été demandé, il ne serait pas exilé en terre étrangère.

Cette première partie, relativement courte, insiste sur l'écoute nécessaire pour connaître la phronêsis et retourner vers le chemin de la sophia, un chemin qu'Israël a abandonné $(3,12)$. On y entend évidemment l'appel de Dt 6,4. Mais à la différence de cet appel lancé au début du Deutéronome, ici il ne s'agit pas d'affirmer d'abord un Credo, mais de choisir entre la vie et la mort. C'est ainsi que résonne un autre appel deutéronomique, celui de Dt 30,15-16: «Vois : je mets devant toi la vie et le bonheur, la mort et le malheur, moi qui te commande aujourd'hui d'aimer YHWH ton Dieu, de suivre ses chemins, de garder ses commandements, ses lois et ses coutumes. Alors tu vivras, tu deviendras nombreux, et YHWH ton Dieu te bénira dans le pays où tu entres pour en prendre possession $»^{17}$. Cet appel à choisir entre le chemin de la vie et celui de la mort, relayé par Jérémie (21,8-9, cf. 17,5-8), résonne clairement pour Baruch : c'est parce qu'Israël a abandonné la source de la sagesse (sophia), Dieu $(3,12)^{18}$, qu'il est en exil, comme compté parmi les morts de l'Hadès $(3,11)^{19}$. Si, au contraire, il avait choisi la route de Dieu et n'avait pas abandonné cette source, il habiterait en paix, pour toujours, dans sa terre $(3,13)$. Ainsi, comme leurs pères, les exilés sont mis face à un choix existentiel majeur. Au vu de la détresse actuelle dans laquelle ils se trouvent, peut-être leur réponse sera-t-elle positive?

17. Trad. ТOB. Significativement, le texte se poursuit en parlant d'écoute, d'idolâtrie et d'exil : "Mais si ton cœur se détourne, si tu n'écoutes pas, si tu te laisses entraîner à te prosterner devant d'autres dieux et à les servir, je vous le déclare aujourd'hui : vous disparaîtrez totalement, vous ne prolongerez pas vos jours sur la terre où tu vas entrer pour en prendre possession en passant le Jourdain » (v. 17-18).

18. Il faut noter qu'il s'agit de la seule fois où cette expression est présente dans la LXX, excepté pour Si 1,5, mais uniquement dans certains manuscrits. Jr 17,13, parle pour sa part de «source de vie» (pêgê zôês).

19. Sur la question des cadavres, on verra aussi Ba 3,4 et Lt Jr 26.31.70 qui leur comparent les idoles. Ceux-ci, comme des cadavres, sont des corps inertes, immobiles et insensibles, absolument à l'opposé du Dieu vivant. C'est l'abandon de celui-ci et de sa Loi qui fait d'Israël un cadavre. Voir aussi Sg 13,10-18. 


\section{Deuxième partie : 3,14-4,1}

La deuxième partie s'ouvre sur un appel tout aussi solennel que le premier. Cette fois, il s'agit d'apprendre où se trouve - où réside - la phronêsis. Car en ce même endroit sont également la force, l'intelligence, la longévité, la vie, la lumière des yeux et la paix - tous conséquences de la phronêsis. De l'apprentissage de celle-ci dépend la connaissance de toutes les autres. On remarquera dans ce cadre la répétition de l'infinitif aoriste gnônai, «pour connaître », déjà présent en $3,9^{20}$. Se dessine ici une identification semblable à celle qu'opérait déjà 3,9 : c'est la connaissance de la sagesse qui conduit à la vie. Là, pour connaître il fallait être très attentif, tendre l'oreille. Ici, il faut apprendre ou peut-être mieux réapprendre. Il s'agirait en effet pour Israël de redécouvrir quelque chose qu'il sait déjà mais qu'il a oublié et qu'il a de ce fait abandonné, ce qui l'a conduit en exil. Il apparaît ainsi que l'écoute dont il est question dans la première partie du poème n'est nullement une écoute passive. Elle demande une participation active du sujet qui passe par l'apprentissage, seul moyen de sortir de cette situation d'exil, de mort.

Ce deuxième appel solennel est relayé, au verset 15, par une question qui évoque "l'inaccessibilité de la sagesse ${ }^{21}$. La réponse à cette question se déploie en deux temps : le premier, situé du côté des humains, est négatif (v. 16-23) ${ }^{22}$ : personne n'a trouvé la sagesse par ses propres forces et sa recherche ; le second, positif, vient un peu plus loin (v.32-37) : le seul qui l'ait trouvée - imaginée, découverte selon le sens de exeuriskô - c'est «celui qui sait toute chose", le Dieu, le seul à être capable de donner la sagesse (v. 37). Ce Dieu n'est autre que «notre Dieu» (v. 36).

Cette réponse en deux temps ménage un contraste saisissant : elle oppose, d'une part, les humains - chefs des Nations qui pensent dominer la création (v. 17), leurs successeurs (v. 20), les marchands et les devins (v. 23), les géants renommés du commencement (v. 26), qui tout en s'étant démenés pour chercher la sagesse ne l'ont pas trouvée - et, d'autre part, Dieu qui l'accorde à qui il veut. Concrètement c'est au peuple qu'il s'est choisi, Jacob / Israël, qu'il l'a donnée. Est ainsi introduite la question de l'élection, sur laquelle on reviendra. Dès lors trouver la sagesse n'est pas seulement ou n'est plus une question de recherche humaine mais de don divin. C'est Dieu en effet qui peut la partager aux humains (v. 37, on y reviendra également).

20. Cet élément pourrait rapprocher structurellement les v. 3 et 14 et faire de ce second verset la conclusion de la première partie.

21. Cette question se retrouve ailleurs dans la littérature de sagesse (cf. Si 1,2-8; Jb 28,12-23) et reçoit toujours la même réponse. Cf. Assan-Dhôte, Moatti-Fine 2008, p. 105.

22. À ce propos Kabasele Mukenge 2008, p. 250-251 écrit : «La partie négative de l'argumentation décrit l'histoire humaine telle qu'elle se laisse saisir en dehors de l'élection divine : c'est une histoire de perdition (cf. v. 19 et 28) ; elle ne donne pas accès à la voie de la connaissance. Le poème décrit cette histoire en remontant, nous semble-t-il, du présent aux origines : d'abord les chefs des nations et leurs descendants actuels; ensuite les nations pré-israélites (celles qui ont précédé Israël au pays de Canaan); enfin les géants primitifs. Aussi loin que l'on puisse remonter, l'humanité sans l'élection divine ne trouve pas la voie de la sagesse. Conséquence : elle est vouée à la mort. Dans un mouvement inverse qui part du Dieu créateur, le poème évoque l'histoire de l'élection : après avoir été le privilège de Dieu, la sagesse a été communiquée à Jacob-Israël. C'est de cette manière seule qu'elle a pu être connue des hommes». 
Avec une pointe d'ironie, le premier temps de la réponse à la question du verset 15 décrit ceux qui n'ont pas découvert la sagesse : les puissants et les riches ne l'ont pas trouvée parce qu'ils sont en réalité bien moins puissants qu'ils ne le croient. Ils ne sont pas "pour toujours", ils sont éphémères, puisqu'ils sont descendus dans l'Hadès (v. 19) - morts comme Israël en exil (cf. v. 11). D'autres après eux ont pris leur place, mais eux non plus n'ont pas trouvé la route de la science (v. 20), que ce soit les Nations ou les chercheurs d'intelligence. Ces derniers ont découvert quelque chose de grand ${ }^{23}$, mais leur trouvaille est restée vaine car ils n'ont pas connu la route de la sophia, c'està-dire la route de Dieu, celle qui mène à lui (v. 23$)^{24}$. Dès lors, bien qu'ils aient trouvé l'intelligence - qui est une forme de sagesse - il leur manque l'essentiel, à savoir la route de la sagesse (sophia, v. 23). Ce n'est qu'un peu plus avant que le lecteur apprendra que seul le Créateur connaît cette route (v. 31-32). C'est lui qui l'a imaginée, tout comme il a imaginé la route de la science, deux routes qu'il a données à Jacob / Israël (v. 37), autrement dit au petit, au faible, plutôt qu'aux géants (aux demi-dieux ?) qui pourtant sont nés dans sa maison (v. 26).

Ainsi, malgré leur statut, c'est à cause de leur stupidité que les grands sont détruits (v. 27) : bien que savants dans certains domaines (de l'élevage à la guerre en passant par le travail de l'or et de l'argent), il leur a manqué la vraie science - voir l'opposition entre les versets 26 et 27 - car ce n'est pas à eux que Dieu a choisi de la donner. C'est le petit, le serviteur, celui qui est aimé de Dieu qui a été choisi pour la recevoir et - c'est probablement ce qu'indique le verset 38 - lui permettre ainsi de résider parmi les humains qui pourront ainsi la voir. Ce verset 38 est souvent considéré comme une glose chrétienne ${ }^{25}$. Pourtant, il a toute sa place dans cette exhortation telle que nous la lisons aujourd'hui ${ }^{26}$. En effet, cette affirmation souligne que l'élection d'Israël est en vue d'une mission à portée universelle. Ainsi, si les deux volets de la réponse à la question du verset 15 construisent une nette opposition entre Israël et les Nations, la sagesse - encore faut-il savoir s'il s'agit de la phronêsis, de la sophia ou de la route de la science - peut être vue, voire connue, de tous, grâce au don que Dieu en fait à son élu.

Dès lors, c'est bien le don fait à Jacob / Israël qui a permis à la sagesse d'être vue sur la terre et de vivre parmi les hommes, comme semble le confirmer l'identification qui est faite immédiatement après entre la sagesse et la Torah de vie (4,1, cf. Si 24,23). Car la Torah est également un don fait à Israël. Ainsi, l'identification entre ces deux dons laisse penser que c'est bien parce qu'il «tient», c'est-à-dire qu'il observe la Loi qu'Israël rend visible la sagesse, car l'observance de la Loi (son écoute et sa mise en pratique) est la sagesse véritable.

23. Leur découverte (cf. 3,18) est «infinie, [elle est] pourtant restée vaine». À cette découverte, s'oppose celle de «Dieu (3,32.37 exeúriskein) de la route de la science », cf. Assan-Dhôte, Moatti-Fine 2008, p. 107.

24. Comme l'écrit Kabasele Mukenge 1998, p. 239 : «L'accumulation des verbes de mouvement [dans ces versets] traduit une recherche assidue, mais qui ne peut aboutir. Le non-aboutissement ne résulte pas du fait qu'on n'a pas essayé de chercher ; il s'inscrit dans l'incapacité fondamentale de "savoir". En même temps, cette démonstration rappelle que la sagesse recherchée par ceux que Dieu n'a pas choisis est trompeuse, apparente ».

25. Cf. Kabasele Mukenge 1998, p. 249-250 et n. 73 pour quelques références bibliographiques à ce propos.

26. Et cela, malgré l'histoire complexe de la transmission de ce texte. 
Cette identification entre la sagesse - mais s'agit-il de la phronêsis, de la sophia ou encore de la route de la science? - et la Loi crée un certain effet de surprise ${ }^{27}$. Mais il est clair que pour Israël, le don de la Torah équivaut à celui de la sagesse. Ainsi l'identification de l'une avec l'autre suggère assez clairement que seule la Torah permet d'atteindre la sagesse ${ }^{28}$. En réalité, cependant, les deux visent une vie bonne, le shalôm pourrait-on dire. De ce fait, elles sont indispensables pour vivre et ne pas mourir. Il est du reste intéressant de remarquer que c'est dans ce contexte, à la fin de cette deuxième partie (v.36), qu'apparaît pour la première fois dans l'exhortation une affirmation, qui résonne comme une déclaration de foi : "c'est lui notre Dieu », c'est-à-dire le Dieu d'Israël, ce que souligne également à sa manière l'autre exclamation en première personne qui vient clore l'exhortation en 4,4 («Heureux sommes-nous, Israël, parce que ce qui plaît à Dieu nous est connu»). Cette affirmation du verset 36 reprend également en écho celle du verset 35, la première de ce type dans notre texte: d'une seule voix les étoiles répondent «nous sommes là» à l'appel du Créateur. Comme elles, Israël, reconnaissant son Dieu et le don qu'il lui a accordé, est appelé à répondre d'une seule voix, par l'écoute, l'apprentissage et le retour à Lui et à la Loi / Sagesse.

\section{Troisième partie : 4,2-4}

Le troisième et dernier appel est tout aussi solennel que les deux premiers. Jacob est appelé à revenir et à se saisir de la sagesse, que ce soit la phronêsis, la sophia ou la route de la science, à moins que ce ne soit les trois ensemble. Autrement dit, Jacob ne doit pas céder aux sirènes de la sagesse vaine et éphémère des vainqueurs, de ceux qui l'ont emmené en exil. Il doit se ressaisir - et se ressaisir de sa Loi $^{29}$ - pour redevenir lui-même, pourrait-on dire. Il faut qu'il s'empare de la sagesse, qu'il la thésaurise et qu'il se mette en route vers sa lumière - mais pour cela il faut qu'il apprenne où elle est (cf. 3,14$)$ et qu'il ne cède pas à d'autres ce qui le rend unique $(4,3)$ : l'élection, l'alliance et la Loi qui la régit.

Comme la deuxième partie du poème, la troisième insiste sur l'élection d'Israël. S'agit-il de l'affirmation d'un particularisme qui conduit non seulement à ne pas se confondre avec les autres, mais aussi à conserver jalousement le privilège reçu? En effet, les termes de cette affirmation peuvent sembler arrogants : affirmer que «nous-Israël» sait ce qui plaît à Dieu est pour le moins l'expression d'une très haute conscience de soi ouvrant la porte à un mépris des autres. Mais ne peut-on comprendre l'élection plus positivement ${ }^{30}$ ? La définition qu'Israël donne de sa propre identité pour la penser sur la base de la Torah et de la sagesse, toutes deux

27. Comme l'écrivent Assan-Dhôte et Moatti-Fine 2008, p. 111, «cette identité de la Loi et de la sagesse, privilège d'Israël, ne se retrouve aussi clairement exprimée qu'en Si 24,23, mais avec un lexique tout à fait différent ».

28. Cela implique évidemment une promesse de restauration pour les exilés à qui s'adresse cette exhortation.

29. Dans ce sens, Assan-Dhôte, Moatti-Fine 2008, p. 112 : ce texte est une «invitation faite à Israël de se ressaisir de sa Loi».

30. Les versets 37 et 38 permettent de le faire également. 
d'origine divine ${ }^{31}$, est en lien profond avec l'alliance. Ainsi, on peut probablement y lire l'expression de la volonté de renouer avec la mission confiée à Abram d'abord (Gn 12,3) et au peuple ensuite (Ex 19,5), d'être médiateur entre YHwh et les Nations. Cette mission s'exprime ici en termes de visibilité du chemin qui mène à Dieu : Israël doit agir de telle sorte qu'il donne à voir ce chemin et qu'ainsi les Nations puissent également s'y engager. Ainsi il est vital pour tous qu'Israël ne cède pas sur ce qui fait qu'il est lui-même, car si de cela dépend sa vie, celle des Nations en dépend aussi.

\section{Reprise de l'ensemble}

Au regard de ce très rapide commentaire, il ne semble pas que l'on puisse parler d'une personnification de la sagesse dans Baruch ${ }^{32}$. Cette exhortation en propose plutôt une définition à travers une série de dévoilements successifs. Ceux-ci touchent aux divers aspects qui la composent et semblent être au nombre de trois. Le premier est plutôt pratique et est représenté par la phronêsis. Le second est plutôt religieux et est représenté par la sophia. Le troisième, la hodos epistêmès, toucherait davantage au politico-social. De ces trois termes, l'un serait plutôt du côté divin et de ce fait inaccessible (sophia), alors que les deux autres seraient plutôt du côté humain et, de ce fait, accessibles. Les trois termes étant des mots féminins, permettent de jouer sur une certaine indétermination entre les niveaux qui touchent aux différents aspects de la vie d'Israël. Chacun d'entre eux est intimement lié à la Torah qui indique le chemin à suivre pour une vie épanouie en alliance, ce qui équivaut très vraisemblablement à la sagesse. Mais cette indétermination, en tout cas grammaticale, donne à penser que les trois niveaux sont imbriqués et qu'il n'est donc pas toujours facile de les distinguer. Ainsi, il est logique qu'écouter, apprendre et observer la Torah est pour Israël la manière concrète de répondre à l'appel qui lui est ici lancé.

Cette indétermination est également soulignée par la multiplicité des chemins à parcourir pour arriver à la sagesse, des routes qui semblent elles aussi se croiser et s'entremêler : «la route de Dieu» (v. 13); «la route de la science» (v. 20.27.37); «la route de la sophia» (v. 23), ou encore «sa route» (v. 30). Une route (de multiples routes) sur laquelle (lesquelles) Jacob doit s'acheminer (v. 42), aussi et dans un premier temps pour revenir de cet exil dans lequel il s'est lui-même engouffré, comme l'affirme Baruch, et avant lui Jérémie.

\section{QUELQUES LIENS AVEC JÉRÉMIE LXX}

Arrivant au livre de Baruch, le lecteur de Jérémie LXX n'est pas du tout dépaysé. Il y retrouve bon nombre de thématiques communes et de résonnances qui lui donnent clairement l'impression qu'en lisant Baruch, il lit en quelque sorte la suite de Jérémie, avec, cette fois, une réponse positive de la part des auditeurs du livre lu (Ba 1,1), là où ceux de Jr 36 s'étaient obstinés dans le refus d'écouter la parole prophétique.

31. Selon Grätz 2013, p. 187-201, ce texte illustre la volonté d'une entité politique à penser et à définir sa propre identité et qui le fait à travers ces deux dons d'origine divine (voir en particulier p. 200).

32. Kabasele Mukenge 1998, p. 270 est du même avis. 
Le premier lien évident entre Jérémie et l'exhortation sapientielle de Baruch est thématique : la question de l'écoute (ou du refus d'écoute) de la parole prophétique ou de la Loi. Cette attitude essentielle demandée aux auditeurs des deux livres fait écho et répond aux multiples appels prophétiques à l'écoute de la parole divine transmise par les prophètes ou contenue dans la Loi. Elle relaie également les multiples accusations des prophètes, en particulier de Jérémie, reprochant au peuple d'avoir refusé d'écouter cette parole $(3,13 ; 7,13.24 \text {, etc. })^{33}$. Le livret de Baruch s'ouvrait sur une confession : «nous n'avons pas écouté la voix du Seigneur notre Dieu» (Ba 1,18 ; cf. 2,10$)$, de sorte que la destruction de Jérusalem et l'exil de ses habitants sont clairement présentés comme la conséquence du refus de la parole, en particulier de celle de Jérémie ${ }^{34}$. Ce sont donc la perversion et les actions mauvaises du peuple qui entraînent l'exil que les auditeurs intradiégétiques du discours de Baruch sont en train de vivre. Ils auraient pu l'éviter s'ils avaient écouté (ou si leurs pères avaient écouté, voir Jr 43 et Ba 1, cf. Jr 11,8) les prophètes envoyés depuis la sortie d'Égypte jusqu'à la génération qui les a précédés (Jr 7,25), de Moïse à Jérémie. Ainsi la raison invoquée pour expliquer la détresse présente est identique en Jérémie et Baruch :

à la question de savoir pourquoi le pays est perdu, incendié comme un désert, Jr 9,12 répond : «parce qu'ils ont abandonné ma Loi » [...]. Cette réponse est similaire à celle que $\mathrm{Ba}$ 3,12 donne à une question semblable: «tu as abandonné la source de la sagesse». Et la suite de l'instruction, on le sait, identifie la sagesse et la Loi $(\mathrm{Ba} 4,1)^{35}$.

Concernant la question de l'écoute de la parole (prophétique et Loi) comme chemin d'acquisition de la sagesse, deux autres rapprochements intéressants et suggestifs peuvent être faits. Le premier concerne la question de l'idolâtrie du peuple. Le second la possibilité d'une vie en paix.

L'idolâtrie du peuple est liée à la fois au manque d'écoute et à la non-connaissance de Yнwн. À maintes reprises, Jérémie affirme que le peuple ne connaît ni YHWH (cf. Jr 4,22; 9,2.5) ni ses ordres (cf. Jr 8,7) et qu'il refuse même de le connaître (cf. Jr 9,5). $\mathrm{Au}$ lieu de se tourner vers la source de la vie (Jr 17,13 et Ba 3,12), Israël se tourne vers les idoles qu'il ne connaît pas et qui ne le connaissent pas, aussi sera-t-il exilé sur une terre inconnue. Pour le dire en d'autres termes, Israël choisit la mort plutôt que la $v^{3}{ }^{36}$. Ainsi, ne pas vouloir connaître YHWH implique également le refus d'une conduite bonne (Jr 7,3), amplement évoquée en Ba 3,9-4,4 par le thème de la route à rechercher et à suivre. Malgré leurs comportements déviants, certains continuent

33. En hébreu, sur 389 usages du verbe shama dans le corpus des Prophètes Seconds, 186 se trouvent chez Jérémie. En grec, le verbe akouô est utilisé 316 fois dans le corpus Is, Jr-LtJr, Éz, XII, dont 137 en Jérémie et 159 dans l'ensemble du corpus jérémien. C'est dire l'importance de la thématique.

34. Il n'y a pas lieu d'insister sur ce point ici. Notons seulement que narrativement le lien est fait entre le livre lu en Ba 1,1 et celui qui le précède immédiatement dans le canon grec: Jérémie. Voir sur ce point Wénin 1999, p. 231-243.

35. Kabasele Mukenge 1998, p. 277-278.

36. Cet élément est mis narrativement en évidence par la mention des cadavres ou des morts qui sont immobiles et sans vie comme les idoles (cf. Jr 2,23 ; Ba 3,4 et LtJr 26.31), mais sont aussi évoqués par le biais des ossements des rois, ainsi que par la mort, choisie par ceux qui préfèrent continuer à ne pas écouter la parole prophétique bien que celle-ci soit en partie déjà réalisée (cf. Jr 7,33-8,3). 
d'affirmer qu'ils sont sages (Jr 8,8) car ils ont la Loi de Yнwн de leur côté ! Mais évidemment, il ne suffit pas d'avoir, de posséder la Loi écrite ${ }^{37}$ pour qu'elle agisse comme par magie. Encore faut-il la pratiquer et la vivre, comme l'affirme Jérémie. Car selon lui, «le faux stylet du scribe l'a faite devenir [la Loi] vaine / futile». Rien d'étonnant donc que «les sages ont été confondus (ou dans la honte), terrifiés et capturés. Car ils ont rejeté la parole de YHwH » (Jr 8,9). Dès lors, Jérémie peut raisonnablement se demander si la sagesse (sophia) est en eux, une question qui ne peut recevoir qu'une réponse négative.

Toujours autour de la question de l'écoute, c'est la possibilité d'une vie paisible qui résonne entre Jérémie et Baruch, autour d'un épisode en apparence banal mais très significatif, celui des Récabites (42 LXX, cf. 35 TM) ; de façon significative, trois mots-clés de ce chapitre se trouvent dans la première partie de l'exhortation à la sagesse. Ces trois mots sont «écouter», les «commandements» (entolê, Ba 3,9 et Jr 42,16.18, seuls usages de ce terme dans l'ensemble du corpus jérémien) et « habiter » (Ba 3,13, Jr 42 passim). En Baruch, au moyen d'un raisonnement par l'absurde, il est dit au peuple exilé que s'il avait suivi la route de Dieu il habiterait en paix pour toujours. Autrement dit, ils vivraient ce que les Récabites vivent, eux dont l'écoute obéissante leur a valu d'habiter à Jérusalem et de se tenir en présence du Seigneur de nombreux jours (Jr 42,19). Voilà qui illustre encore une fois les choix négatifs du peuple qui avait tout ce qu'il lui fallait pour bien vivre, mais qui a rejeté la parole de vie.

\section{POUR NE PAS CONCLURE...}

On pourrait évidemment continuer la liste des rapprochements entre le passage ici analysé et Jérémie LXX. Ce qui est rapidement évoqué ici suffit, cependant, me semble-t-il, à montrer que, d'un point de vue synchronique, on peut constater un mouvement d'ensemble qui commence en Jérémie et aboutit en Baruch. Cela dit, il faut noter une inversion de taille : elle porte sur le vocabulaire utilisé pour parler de la sagesse. La phronêsis n'intervient qu'une seule fois en Jérémie $(10,12)$ et caractérise la sagesse du Dieu créateur - tout comme la sophia en 28,15 (//51,51 TM). Jérémie LXX évoque aussi une sagesse plus générale que Baruch nommerait peutêtre phronêsis (Jr 30,1 // 49,7 TM). L'adjectif correspondant se réfère à des humains (peuple, ceux qui se croient sages) et sert plutôt à les dénoncer ou les railler (cf. Jr 8,9). Il est évident qu'on pourrait conclure de ces observations que les termes utilisés par les auteurs de ces livres bibliques (Jérémie et Baruch) peuvent être considérés comme synonymes ou qu'ils le sont devenus dans l'évolution de la langue. Lisant

37. Kabasele Mukenge 1998, écrit à ce propos p. 279-280 : «Dans l'instruction sapientielle de Baruch, la distinction entre une prétendue sagesse et la sagesse véritable ne se fait pas à l'intérieur du peuple d'Israël, mais à l'égard des autres nations $(3,16.22-23)$, ou plus précisément, d'un peuple étranger $(4,3)$. D’un côté, Israël qui, à travers la Loi écrite, a reçu la sagesse ; de l'autre, les autres peuples qui, malgré de vaines tentatives, ne l'ont pas trouvée. Certes, pour Baruch comme pour Jérémie, la sagesse ne consiste pas seulement à posséder la Loi écrite. Les appels lancés à Israël-Jacob, au début et à la fin de l'instruction sapientielle, montrent la même insistance sur l'écoute et l'obéissance de la Loi. Si relecture il y a, l'instruction sapientielle installe dorénavant une autre polémique : entre une sagesse apparente partagée par les nations et attirante pour Israël, et la sagesse véritable donnée au seul Israël». 
Jérémie LXX, le lecteur peut effectivement avoir cette impression. Mais à cela Baruch ajoute une certaine complexité, soulignant cette indétermination des termes que Jérémie LXX suggérait déjà. Comprendre la sagesse et ce qu'elle revêt n'est pas simple, car la notion même de sagesse est complexe et couvre chaque aspect de la vie humaine, singulièrement et collectivement. Ainsi, selon le prophète et son fidèle secrétaire, c'est suivre la Torah qui est et reste la meilleure manière d'y parvenir.

\section{BIBLIOGRAPHIE}

Assan-DhôTe (I.), MoATti-Fine (J.)

2008 Baruch, Lamentations, Lettre de Jérémie (La Bible d'Alexandrie, 25/2), Paris.

BOGAERT (P.-M.)

1974 «Le nom de Baruch dans la littérature pseudépigraphique : l'apocalypse syriaque et le livre deutérocanonique», dans W.C. Van Unnik (dir.), La littérature juive entre Tenach et Mischna. Quelques problèmes (Recherches Bibliques, 9), Leyde, p. 56-72.

1982 «Le personnage de Baruch et l'histoire du livre de Jérémie. Aux origines du Livre deutérocanonique de Baruch», dans E. A. Livingstone (dir.), Studia Evangelica, VII. Papers presented to the Fifth International Congress on Biblical Studies held at Oxford, 1973 (Texte und Untersuchungen zur Geschichte der Altchristlichen Literatur, 126), Berlin, p. 73-81.

BURKES (S.)

1999 "Wisdom and Law: Choosing Life in Ben Sira and Baruch", Journal for the Study of Judaism in the Persian, Hellenistic, and Roman Period 30, p. 253-276 [En ligne : http://www.jstor.org/stable/24668456].

GRÄTZ (S.)

2013 " "Wisdom" and "Torah" in the Book of Baruch", dans B.U. Schipper, D. A. Teeter (dir.), Wisdom and Torah : The Reception of "Torah" in the Wisdom Literature of the Second Temple Period, Leyde, p. 187-201.

HARRELSON (W.)

1992 «Wisdom Hidden and Revealed according to Baruch - Baruch 3,9-4,4», dans E. Ulrich, J. W. Wright, R.P. Carroll, P. R. Davies (dir.), Priests, Prophets and Scribes. Essays on the Formation and Heritage of Second Temple Judaism in Honour of Joseph Blenkinsopp (Journal for the Study of the Old Testament. Supplement series, 149), Sheffield, p. 158-171.

KABASElE MUKenge (A.)

1998 L'unité littéraire du livre de Baruch (Études Bibliques. N.S., 38), Paris.

WÉNIN (A.)

1999 "Y a-t-il un "livre de Baruch" ? À propos du livre récent d'André Kabasele Mukenge », dans J.-M. Auwers, A. Wénin (dir.), Lectures et relectures de la Bible. Festschrift P.-M. Bogaert (Bibliotheca ephemeridum theologicarum Iovaniensium, 144), Louvain, p. 231-243. 